\title{
On Validating Boolean Optimizers
}

\author{
Antonio Morgado ${ }^{1}$ and Joao Marques-Silva ${ }^{1,2}$ \\ 1 CSI/CASL,UCD, Dublin, Ireland \\ 2 IST/INESC-ID, TUL, Lisbon, Portugal \\ \{ajrm,jpms\}@ucd.ie
}

\begin{abstract}
Boolean optimization finds a wide range of application domains, that motivated a number of different organizations of Boolean optimizers since the mid 90s. Some of the most successful approaches are based on iterative calls to an NP oracle, using either linear search, binary search or the identification of unsatisfiable sub-formulas. The increasing use of Boolean optimizers in practical settings raises the question of confidence in computed results. For example, the issue of confidence is paramount in safety critical settings. One way of increasing the confidence of the results computed by Boolean optimizers is to develop techniques for validating the results. Recent work studied the validation of Boolean optimizers based on branch-and-bound search 18[17. This paper complements existing work, and develops methods for validating Boolean optimizers that are based on iterative calls to an NP oracle. This entails implementing solutions for validating both satisfiable and unsatisfiable answers from the NP oracle. The work described in this paper can be applied to a wide range of Boolean optimizers, that find application in Pseudo-Boolean Optimization and in Maximum Satisfiability. Preliminary experimental results indicate that the impact of the proposed method in overall performance is negligible.
\end{abstract}

Keywords: Boolean Optimization, Proof Traces

\section{Introduction}

The remarkable advances of Boolean Satisfiability (SAT) algorithms in past years, motivated their widespread use in many practical applications. Moreover, some applications require the Boolean algorithms to optimize some cost function (e.g. [16, 29], 28, [20]).

These practical applications depend on correct results computed by Boolean optimizers to fulfill their objective. One way of increasing the confidence in the results computed, is to develop techniques for validating the results. In the context of (pure) SAT, validation of unsatisfiable answers has been addressed by Zhang \& Malik [32, whereas the validation of satisfiable answers corresponds just to a check if the returned assignment satisfies all the clauses. Certified validation of SAT has been considered in [19].

Validation of Boolean optimizers has been studied recently [1817]. However, this work covers only Boolean optimizers that are based on branch-and-bound 
search, e.g. BSOLO [21. Nevertheless, many state of the art Boolean optimizers are based on iterative calls to a SAT solver. For example, minisat+ 9, sat4j [6] (-pb,-maxsat), PBS [1, pueblo [30, msuncore [24|23|22], PM2 [4, WPM1 [4, WMSU1 22].

This paper develops methods for validating the results computed by Boolean optimizers, that are based on iterative calls to a SAT solver. The idea is to allow for an independent checker to receive the information returned by the Boolean optimizers and validate the result. The paper shows that, to improve the efficiency of the solution checking process, it is unnecessary for the checker to validate all the iterations of the solver, which can be as many as the number of clauses. This result is general and, as the paper shows, holds for most Boolean optimization algorithms based on iterative calls to a SAT solver. Moreover, the paper shows that, similarly to SAT, the time to check MaxSAT solutions is in general negligible when compared with the time the algorithms take to compute the optimum solution.

The paper is organized as follows. Section 2 introduces Boolean optimization problems and describes three iterative approaches currently used by Boolean optimizers. Section 3 presents our methods of validation of the results returned. Experimental results with our methods of validation are shown in Section 4 , and the paper concludes in Section 5.

\section{Preliminaries}

This section describes Boolean optimization problems and the notation used in the paper. First, Boolean optimization problems are introduced, followed by a brief introduction to the different iterative algorithms to solve Boolean optimization problems. Familiarity with the basic concepts of Boolean variables, literals, clauses, conjunctive normal formula (CNF), etc, is assumed, but additional detail can be found for example in 7. A CNF formula can be viewed either as a conjunction of clauses or as a set of clauses. Similarly, a clause can be viewed as a disjunction of literals or as a set of literals.

The Boolean optimization problems described in this section can be seen as optimization extensions of the propositional satisfiability problem (SAT). The SAT problem is the problem that, given a CNF formula $\varphi$ on a set of Boolean variables, determines one assignment $\sigma$ to the variables such that $\sigma$ satisfies $\varphi$, if such $\sigma$ exists. Otherwise UNSATISFIABLE is reported.

This work considers three different Boolean optimization problems. The MinCost SAT problem [12], the Pseudo-Boolean optimization problem (PBO) [28] and the Maximum Satisfiability problem (MaxSAT) 20. The MinCost SAT problem has as input a CNF formula $\varphi \equiv \bigwedge_{j=1}^{m} \omega_{j}$, and a cost function represented as in Equation (10), where each $c_{i}$ is a constant and literal $l_{i}$ is either variable $x_{i}$ or the negated variable $\overline{x_{i}}$. The goal in MinCost SAT is to determine a satisfying assignment $\sigma$ for $\varphi$ that minimizes the value of the cost function.

$$
\min : \sum_{i=1}^{n} c_{i} a_{i} \text { where }\left\{\begin{array}{l}
a_{j}=1 \text { if } \sigma\left(l_{i}\right)=\text { true } \\
a_{j}=0 \text { otherwise }
\end{array}\right.
$$


The Pseudo-Boolean Optimization problem (PBO) also considers a cost function (also called the objective function), but $\mathrm{PBO}$ does not consider the formula $\varphi$ to be in $\mathrm{CNF}$ format. Instead in $\mathrm{PBO}$, the constraints are linear 3 inequations of pseudo-Boolean variables, that is, the variables have either value 0 or 1 . An instance of PBO can be formulated as in Equation (2).

$$
\begin{array}{cl}
\min : \sum_{i=1}^{n} c_{i} x_{i} & 1 \leq j \leq m \\
& a_{i, j}, b_{j}, c_{i} \in \mathbb{Z} \\
\text { s.t. } \sum_{i=1}^{n} a_{i, j} l_{i, j} \leq b_{j} & l_{i}, l_{i, j} \in\left\{x_{i}, \overline{x_{i}}\right\} \\
& x_{i} \in\{1,0\}
\end{array}
$$

The last Boolean Optimization problem considered is the Maximum Satisfiability (MaxSAT) problem which given a CNF formula $\varphi \equiv \bigwedge_{j=1}^{m} \omega_{j}$ consists on identifying an assignment $\sigma$ that satisfies the maximum number of clauses of $\varphi$. MaxSAT can be represented as in Equation 3. Typically in MaxSAT the given formula $\varphi$ is unsatisfiable (otherwise MaxSAT corresponds to the SAT problem). Note also that despite the MaxSAT problem being defined in terms of the maximum number of satisfied clauses, current MaxSAT solver report as solution the minimum number of unsatisfied clause 4 . The maximum number of satisfied clauses is then obtained by subtracting from the total number of clauses, the minimum number of unsatisfied clauses.

$$
\max : \sum_{j=1}^{m} a_{j}, \quad\left\{\begin{array}{l}
a_{j}=1 \text { if } \sigma\left(\omega_{j}\right)=\text { true } \\
a_{j}=0 \text { otherwise }
\end{array}\right.
$$

Several variants of MaxSAT can be considered, namely partial MaxSAT, weighted MaxSAT and weighted partial MaxSAT. In partial MaxSAT some of the clauses on $\varphi$, called the hard clauses, must be satisfied by $\sigma$. By opposition, the clauses of $\varphi$ which may or may not be satisfied by $\sigma$ are called soft clauses. Thus, the objective in partial MaxSAT is to determine $\sigma$ that satisfies all the hard clauses of $\varphi$, and maximizes the number of satisfied soft clauses of $\varphi$, if such $\sigma$ exists. If no $\sigma$ is able to satisfy the hard clauses of $\varphi$, then UNSATISFIABLE is returned.

In weighted MaxSAT each clause is associated to a weight that represents the cost of satisfying the clause. The objective is to maximize the sum of the weights of the satisfied clauses. Weighted partial MaxSAT combines the previous two. The set of clauses is divided in hard and soft clauses, and the soft clauses are associated with weights. The objective is to maximize the sum of the weights of the satisfied soft clauses while satisfying all the hard clauses.

This paper focus on (pure) MaxSAT and partial MaxSAT.

\footnotetext{
${ }^{3}$ Some of the current work in PBO does not require the constraints or the objective function to be linear. The linear formulation is preferred for readability, but nonlinear constraints or non-linear objective function can be considered.

${ }^{4}$ In the pseudo-codes presented it is the minimum number of unsatisfied clauses that is returned.
} 
Although the Boolean optimization problems have different optimization objectives and different formalisms, it is possible to translate an instance of one problem into the others $3 / 2 \mid 1422$.

Despite only considering these Boolean optimization problems, the methods proposed in this paper for checking computed results can be adapted to other Boolean optimization problems, such as MaxSMT 26 8], MaxASP 27] or Weighted Boolean Optimization (WBO) 22.

\subsection{Boolean Optimization Algorithms}

In this section we outline three approaches to solve Boolean optimization problems for which in the next section we apply our method of validation. The approaches are based on linear search, binary search and (unsatisfiable) core guided search. Given that translations between the Boolean optimization problems are known, in this section, we concentrate on the problem of MaxSAT for presenting the different algorithms.

The idea of the algorithms presented is to start with a bound for the optimum value and create a CNF instance that improves over the given bound. Depending on the satisfiability of the created CNF instance, the bound is updated and the process is restarted until the optimum value is found.

Considering the case of MaxSAT, the bound corresponds to the number of clauses that are unsatisfiable. The optimum value is then obtained by subtracting the bound from the total number of clauses. The algorithms consider integer variables $\mu / \lambda$ to hold the value of the upper/lower bound (respectively) depending on the type of search.

All the three approaches consider relaxation variables which are new fresh variables that are added to the clauses of the MaxSAT instance. If $r_{i}$ is the relaxation variable associated with clause $\omega_{i}$, then $r_{i}$ is assigned to true if $\omega_{i}$ is unsatisfiable. Otherwise, $r_{i}$ is assigned to false.

Linear Search The algorithms described in this section perform a linear search on the optimum value. The use of Linear search for MaxSAT was first proposed in 2006 [1].

A new working CNF formula $\varphi_{W}$ is created to contain all the clauses of the form $\left(\omega_{i} \vee r_{i}\right)$, where $\omega_{i}$ is a clause of the MaxSAT instance and $r_{i}$ is the relaxation variable associated to $\omega_{i}$. Each iteration adds a cardinality constraint to $\varphi_{W}$ to constrain the number of relaxation variables assigned to true to be at most a given value. The exact value used in the cardinality is dependent on the type of search. Two types of linear search are possible. Either searching through unsatisfiable CNF instances or searching through satisfiable CNF instances. The case of searching through unsatisfiable CNF instances is called Linear Search unsatisfiable-satisfiable, and the algorithm starts by seting a lower bound $\lambda$ to 0 . Each iteration for which an unsatisfiable instance is found, $\lambda$ is increased by one. The search proceeds until a satisfied instance is found. The pseudo-code is presented in Algorithm 1 . 


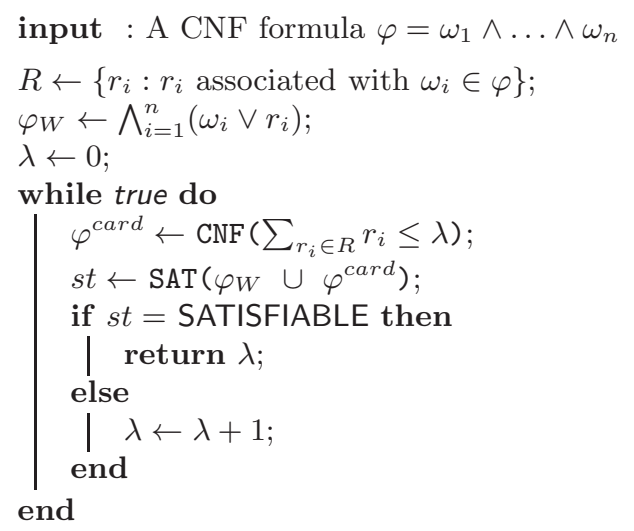

Algorithm 1: Linear Search unsatisfiable-satisfiable for MaxSAT

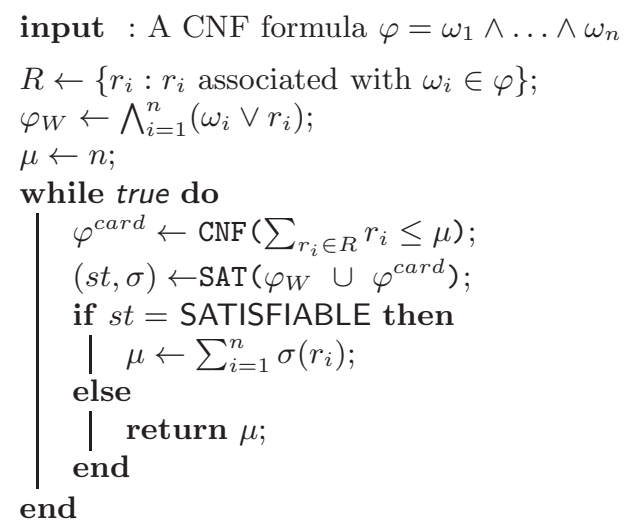

Algorithm 2: Linear Search satisfiable-unsatisfiable for MaxSAT

The case of searching through satisfiable CNF instances is called Linear Search satisfiable-unsatisfiable. The algorithm uses an upper bound $\mu$ which initially is set to the total number of clauses. Each iteration, for which $\varphi_{W}$ is found satisfiable, decreases $\mu$ by the number of relaxation variables assigned to true. The number of relaxation variables assigned to true is obtained through the assignment $\sigma$ returned by the SAT solver on satisfiable instances.

The search stops when an unsatisfiable instance is found. The pseudo-code is presented in Algorithm 2. An example of a MaxSAT solver that uses Linear Search satisfiable-unsatisfiable is SAT4j-maxsat [6].

Binary Search The algorithm presented in this section is similar to the Linear search algorithms, but making instead a binary search on the value of the bound.

Binary Search uses both an upper bound $\mu$ and and a lower bound $\lambda$, and iteratively creates and solves a CNF instance that includes a constraint relating the cost function with a middle value $\tau$. 


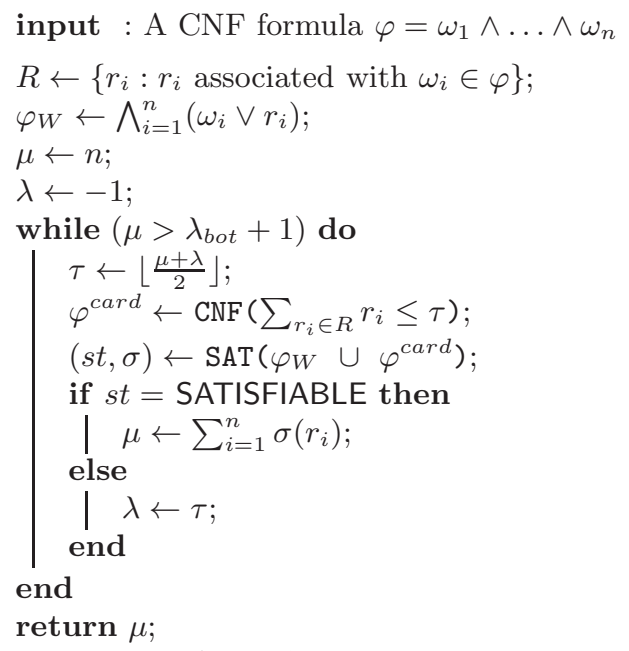

Algorithm 3: Binary search for MaxSAT

The algorithm is shown in Algorithm 3, The relaxation variables and the working formula are created as in the previous linear algorithms. In each iteration the middle value of $[\lambda, \mu]$ is assigned to $\tau$ and the set of clauses that constrains the sum of the relaxation variables to be at most $\tau$ is feed to the SAT solver, together with the working formula. If the SAT solver returns SATISFIABLE, then $\mu$ is updated to the number of relaxation variables assigned to true by the assignment $\sigma$ returned by the SAT solver. Otherwise, $\lambda$ is updated to $\tau$. The search stops when $\mu$ and $\lambda$ differ in one unit, in which case, the maximum number of satisfiable clauses is the number of original MaxSAT clauses minus $\mu$.

The use of binary search for PBO has been discussed 77. Moreover, binary search has been recently used for solving Boolean optimization problems in the context of SMT [8], where the authors developed a theory of $\operatorname{costs} \operatorname{SMT}(\mathcal{C})$ and $\operatorname{SMT}(\mathcal{C} \cup \mathcal{T})$, and propose to solve PBO, MaxSAT and MaxSMT by encoding the problems into $\operatorname{SMT}(\mathcal{C})$ (and $\operatorname{SMT}(\mathcal{C} \cup \mathcal{T}$ ) for MaxSMT).

Core Guided Search Another type of search used for Boolean optimization is based on the generation of unsatisfiable cores. An unsatisfiable core (or simply core) is a sub-formula of the original CNF formula that is unsatisfiable 32 . Current SAT solvers are able to return cores for unsatisfiable instances (which are regarded as a reason for the unsatisfiability of the instance).

The use of unsatisfiable cores for solving (partial) MaxSAT was first proposed in 2006 [1] with the MSU1.0 algorithm. The idea of MSU1.0 is to iteratively eliminate unsatisfiable cores of the problem instance, computed by a SAT solver, by adding new relaxation variables to the clauses and add new constraints to constrain the sum of these relaxation variable to be equal to one.

Recently, several new MSU algorithms [23|24], PM2 algorithm [4], WPM2 [5], bin-core and bin-core-disjoint [15] have been proposed. The differences of the 


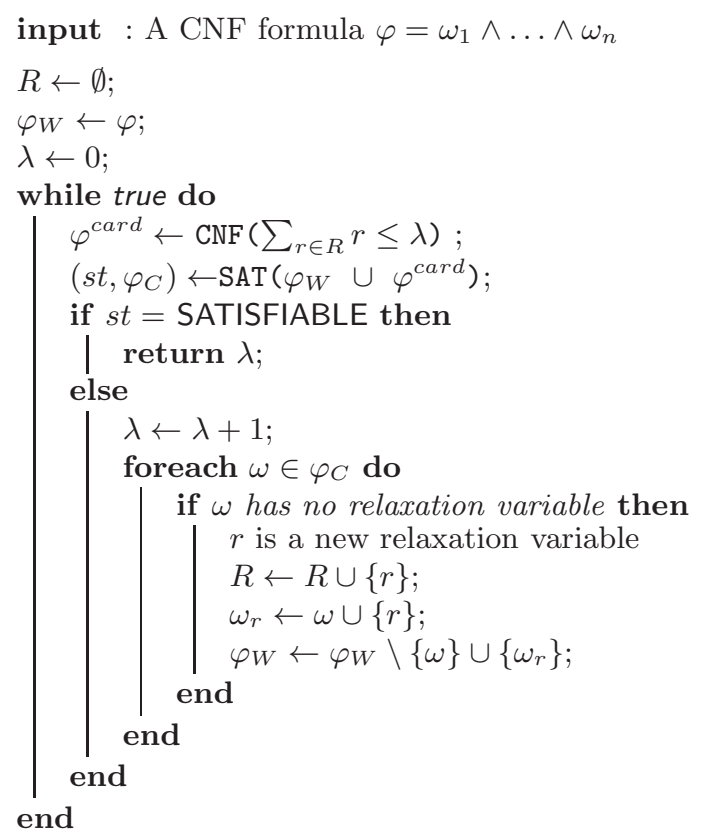

Algorithm 4: Simplified MSU3

algorithms include the number of cardinality constraints used, the encoding of the cardinality constraints, the number of relaxation variables considered for each clause and how the algorithm proceeds (despite being all based on the generation of cores).

In the following we consider a representative algorithm for this class of algorithms. We consider a simplified version of the MSU3 24 algorithm which is depicted in Algorithm 4 . Instead of relaxing all the (soft) clauses as in previous algorithms, the set of clauses that are allowed to be relaxed in one iteration is dependent on the cores reported in the previous iterations. Initially, no clause is allowed to be relaxed, thus the set of all relaxed variables $R$ is empty and the working formula $\varphi_{W}$ is the same as the original formula.

In each iteration the SAT solver is called with the working formula and an AtMost constraint on the number of relaxed variables. If the SAT solver returns UNSATISFIABLE then a new core $\varphi_{C}$ is available. $\lambda$ is increased by one and each original (soft) clause in the core receives a new relaxation variable, if it does not have one yet. Otherwise, the optimum has been found.

\section{$3 \quad$ Validating Boolean Optimizers}

Validating the results provided by a solver is a recurrent problem in applications that rely on correct results for their operation (e.g. 16|29]). If a SAT solver returns an assignment and reports it to be SATISFIABLE, then to check if the 
assignment is indeed a satisfying assignment, it is enough to go through each clause, and check if the clause is satisfied by the assignment. If all the clauses are satisfied, then the assignment is a satisfying assignment. Otherwise, the result is incorrect.

Similarly, it is necessary to validate UNSATISFIABLE outcomes. In 2003, Zhang \& Malik 32 proposed an independent resolution based checker that takes the trace produced by the solver and checks the correctness of the result. Goldberg et al. [13] considers a procedure for the verification of unsatisfiable formulas and in 2009 Weber et al. 31] proposed the use a HOL theorem prover to verify the proofs of unsatisfiability given by minisat [10] and zchaff [25].

In the context of Boolean optimization, the solver needs to provide the optimum value it has found and certificates that the value is correct. Recently, Larrosa et al. 1817] showed how to generate proofs of optimality for branchand-bound procedures that corresponds to a lower-bound certificate and a model to the optimization problem.

This section shows how to modify the algorithms described in the previous section, so that their results can be validated. The objective is to instrument Boolean optimizers to return a uniform certificate with the minimal information, that allows an independent checker to validate the result. Two methods of validation are proposed.

\subsection{Method 1}

All the algorithms of the previous section are based in iteratively searching through CNF instances, either satisfiable or unsatisfiable. The value being optimized is encoded in the CNF instances. The first method to validate the result returned by these algorithms is to consider the value encoded and the results returned by the SAT solver for all iterations, that is, to validate the result returned by the SAT solver for each iteration.

For validation of iterations with satisfiable CNF instances, the solvers report the value that is being tested and the satisfiable assignment returned by the SAT solver. For example, consider the Linear search algorithm going through unsatisfiable instances of the previous section and an instance $\varphi$. Suppose that the algorithm runs for three iterations (all with unsatisfiable results) and that on the fourth iteration the SAT solver reports the instance to be satisfiable. The algorithm would report for the fourth iteration the bound $\lambda=3$ meaning that the total number of satisfied clauses is $|\varphi|-3$, together with the satisfying assignment. In this case, the check needs only to verify that there are exactly three clauses that are not satisfied by the assignment reported.

For the case of unsatisfiable CNF instances, the Boolean optimizers need only to report the trace produced by the SAT solver (as in the case of checking unsatisfiability of SAT solvers [32]).

An independent checker receives the information of the satisfiable iterations, which we call the satisfiable certificates, and the traces of the unsatisfiable iterations (the unsatisfiable certificates), and validates the result. The checker verifies that the satisfiable certificates are correct, that is, the assignment is a satisfiable 
assignment and satisfies the value reported. For the unsatisfiable certificates the checker proceeds as current resolution checkers of SAT solvers in unsatisfiable instances as in Zhang \& Malik [32].

Example 1. Consider the following partial MaxSAT formula:

$$
\begin{aligned}
\text { Soft CLauses: } & \left(\neg x_{1}\right)\left(\neg x_{2}\right)\left(\neg x_{3}\right) \\
& \left(\neg x_{4}\right)\left(\neg x_{5}\right) \\
\text { Hard Clauses: } & \left(x_{1} \vee x_{2}\right)\left(x_{2} \vee x_{3}\right) \\
& \left(x_{3} \vee x_{4}\right)\left(x_{4} \vee x_{5}\right) \\
& \left(x_{1} \vee x_{5}\right)
\end{aligned}
$$

Consider for the example a Linear search algorithm. A correct Linear search algorithm going through unsatisfiable instances would start by relaxing the five soft clauses and then perform four iterations. The first three iterations (with $\lambda=0,1,2)$ would each report an unsatisfiable certificate, while the last iteration (with $\lambda=3$ ) would report a satisfiable certificate.

The checker using the previous method 1 would have to validate the four certificates.

\subsection{Method 2}

Given that the iterations of the approaches described for Boolean optimization converge to the optimum value through satisfiable and unsatisfiable instances, then in the second method of validation, not all the certificates of all the iterations are checked. In fact, the checker needs only to validate the last satisfiable iteration and the last unsatisfiable trace produced. This can easily be seen for the Linear Search algorithms of Section 2.1. Consider a run of the Linear search algorithm moving through unsatisfiable instances. In terms of the variable $\lambda$ and the status returned by the SAT solver st, the run of the Linear search algorithm looks like the following:

$$
\begin{aligned}
& \lambda=0 \quad \text { st }: \text { UNSATISFIABLE; } \\
& \cdots \\
& \lambda=k-1 \text { st }: \text { UNSATISFIABLE; } \\
& \lambda=k \quad \text { st }: \text { SATISFIABLE }
\end{aligned}
$$

The last unsatisfiable iteration has $\lambda=k-1$. If we check that the SAT solver returned the correct unsatisfiable result for the formula $\varphi_{W} \cup C N F\left(\sum_{r \in R} r \leq\right.$ $k-1$ ) of the last unsatisfiable iteration, then we are assured that any of the previous iterations with $\lambda<k-1$ are all unsatisfiable. This is true because the formula $\varphi_{W}$ and the set of relaxation variables remains the same between iterations, and thus the set of solutions of the constraint $\left(\sum_{r \in R} r \leq \lambda\right)$, with $\lambda<k-1$, is included in the set of solutions of the constraint $\left(\sum_{r \in R} r \leq k-1\right)$. Only one satisfiable iteration exists with $\lambda=k$, which corresponds to the optimal value. 
In the case of the Linear search algorithm going through satisfiable instances, it is also easy to demonstrate that it is enough to check the certificates of to the last satisfiable and the last unsatisfiable iterations. Consider a run of the Linear search algorithm going through satisfiable instances. As before, we consider the iterations in terms of the variable $\mu$ and the status returned by the SAT solver st. Consider w.l.o.g. the worst case scenario where the assignment returned $\sigma$ always decreases $\mu$ by one unit. Then a run of the Linear search algorithm (through satisfiable instances) looks like the following:

$$
\begin{aligned}
& \mu=n \\
& \cdots \\
& \mu=n-k+1 \text { st }: \text { SATISFIABLE; } \\
& \mu=n-k \quad \text { st }: \text { UNSATISFIABLE }
\end{aligned}
$$

There is only one unsatisfiable iteration with $\mu=n-k$ and it corresponds to the last unsatisfiable certificate.

The last satisfiable iteration has $\mu=n-k+1$ (the optimum value). If we check that the SAT solver returned the correct satisfiable result for the formula $\varphi_{W} \cup C N F\left(\sum_{r \in R} r \leq n-k+1\right)$ of the last satisfiable iteration, then we are assured that for greater values of $\mu$, the formula is still satisfiable. The reasons for this are analogous to the previous case of Linear search going through unsatisfiable instances.

The case of the Binary search algorithm of Section 2.1 is similar to the Linear search algorithms but using $\mu$ and $\lambda$. The algorithm terminates with $\mu=\lambda+1$, and as in the Linear search algorithms the last unsatisfiable and the last satisfiable iterations will subsume the other iterations, for analogous reasons.

Example 2. Consider one more time the instance of the previous Example 1

A checker using method 2 for validating would not have to validate all the unsatisfiable certificates. Instead, using method 2 would save the checker from validating the first two unsatisfiable certificates. Only the last unsatisfiable certificate and the satisfiable certificate would have to be validated.

An interesting case for validating is the case of the simplified MSU3 algorithm, which changes its set of relaxation variables while it is changing the bound $\lambda$. Due to the change of the set of relaxation variables it is not possible to consider only the last satisfiable certificate and the last unsatisfiable certificate as in the other algorithms.

For example, consider the MaxSAT instance $\{(x) \wedge()\}$ which has an optimum value of $\lambda=1$. Consider also a buggy simplified MSU3 solver with the following run:

$$
\begin{aligned}
& \lambda=0 \text { st }: \text { UNSATISFIABLE core }:\{(x)\} ; \\
& \lambda=1 \text { st }: \text { UNSATISFIABLE core }:\{()\} ; \\
& \lambda=2 \text { st }: \text { SATISFIABLE } \quad \sigma=\left\{x=r_{1}=r_{2}=1\right\}
\end{aligned}
$$

On the first iteration, the solver correctly returns UNSATISFIABLE but with the wrong core $\{(x)\}$. Clause $(x)$ is augmented with the relaxation variable 
$r_{1}$ being transformed into $\left(x \vee r_{1}\right)$. Thus on the second iteration the solver tests the satisfiability of the CNF instance $\left\{\left(x \vee r_{1}\right) \wedge() \wedge C N F\left(r_{1} \leq 1\right)\right\}$, and returns UNSATISFIABLE with the core $\{()\}$. Clause () is relaxed with relaxation variable $r_{2}$ which becomes $\left(r_{2}\right)$. Finally on the third iteration the solver tests the satisfiability of the instance $\left\{\left(x \vee r_{1}\right) \wedge\left(r_{2}\right) \wedge C N F\left(r_{1}+r_{2} \leq 2\right)\right\}$, and reports SATISFIABLE with an assignment $\sigma=\left\{x=r_{1}=r_{2}=1\right\}$ and $\lambda=2$.

The last unsatisfiable certificate, and the last satisfiable certificate are both correct and yet the result is wrong. This example shows that when modifying the set of relaxation variables, it is not enough to check the last two certificates. An additional test is required, to test that among all possible (soft) clauses to relax, the value reported is minimal. This can be achieved by creating a new instance with all (soft) clauses relaxed together with a new constraint that encodes the sum of all relaxation variables being strictly smaller than the result returned by the Boolean optimization solver.

In the previous example the checker would validate both the last satisfiable and unsatisfiable certificates and make the test that the CNF instance $\{(x \vee$ $\left.\left.r_{1}\right) \wedge\left(r_{2}\right) \wedge C N F\left(r_{1}+r_{2}<2\right)\right\}$ is unsatisfiable. The SAT solver would report the instance to be satisfiable, with the satisfying assignment $\left\{x=r_{2}=1, r_{1}=0\right\}$, and the checker would report the MaxSAT solver to have an incorrect result.

Notice that we could have restricted the original satisfying assignment $\sigma$ (reported on the satisfiable certificate) to an assignment $\sigma^{\prime}$ containing only assignments to the original variables $\left(\sigma^{\prime}=\{x=1\}\right)$ and then counted the number of clauses which are not satisfied by original variables. The obtained value could then be compared with the reported $\lambda$. For the previous example we would have obtained that only one clause is unsatisfied, thus realizing that the reported $\lambda=2$ is not minimal. But this is not always the case, if the reported $\sigma$ was instead $\sigma=\left\{x=0, r_{1}=r_{2}=1\right\}$, then the number of clauses not satisfied by original variables would also be two as $\lambda$, thus still requiring the additional test to verify that the reported $\lambda$ is minimal.

The correctness of the second method of validation can be summarized in the following propositions.

Proposition 1 (Validation of Linear/Binary Search).

The result of a Boolean Optimizer based on Linear search or on Binary search is correct if and only if the last unsatisfiable certificate and the last satisfiable certificate are validated.

Proposition 2 (Validation of the Simplified MSU3).

The result of the simplified MSU3 algorithm is correct if and only if the last unsatisfiable certificate (of the modified problem instance) and the last satisfiable certificate are validated together with the validation that the value returned is minimal.

\section{Experimental Results}

This section presents experimental results on checking MaxSAT solutions computed with Binary Search algorithm. The methods outlined in the paper could 


\begin{tabular}{|c|c|c|c|c|c|}
\hline Instance & Opt. Value & Bin Search & Bin Search-Gd & Check All & Check One \\
\hline \hline simp-unif-100_100.09.wcnf & 26 & 1.58 & 1.67 & 0.17 & 0.01 \\
\hline simp-ibd_50.03.wcnf & 54 & 6.04 & 6.62 & 1.34 & 0.01 \\
\hline normalized-aim-100-1_6-yes1-1.wcnf & 100 & 2.07 & 2.12 & 0.02 & 0.02 \\
\hline normalized-aim-200-1_6-yes1-2.wcnf & 200 & 11.28 & 11.34 & 0.04 & 0.04 \\
\hline normalized-ii8a1.wcnf & 54 & 37.07 & 43.15 & 23.42 & 0.07 \\
\hline normalized-jnh1.wcnf & 92 & 8.39 & 9.25 & 2.05 & 0.07 \\
\hline normalized-jnh213.wcnf & 92 & 3.02 & 3.11 & 0.31 & 0.02 \\
\hline normalized-jnh7.wcnf & 89 & 3.95 & 4.23 & 0.76 & 0.01 \\
\hline normalized-par8-1.wcnf & 350 & 41.48 & 41.47 & 0.22 & 0.22 \\
\hline
\end{tabular}

Table 1. Run and checking times for the Binary Search algorithm

be used with the other Boolean optimization approaches studied in this paper. Two types of results are presented. The first type of results show the CPU times of the solver with and without the generation of the certificates. The second type of results concentrate on checking all the certificates versus checking just one certificate. All the results obtained are for the Binary Search algorithm described in Section 2.1. The nine instances used in the results were obtained from the 2009 MaxSAT evaluation, and represent partial MaxSAT instances. A Mac Pro server with 8 GByte of physical memory and a $2.93 \mathrm{GHz}$ processor was used for the experiments. All run times are in seconds.

The values in the columns Bin Search and Bin Search-GC of Table 1 show the running times of the two versions of the solver, with and without the generation of certificates. The table also shows the optimum value for each problem instance. As can be concluded, there can be a difference in run times between generating and not generating certificates. Similar conclusions were observed in 1817.

The values in the columns Check ALL and Check One of Table 1 represent the running times of checking all the unsatisfiability certificates, and of checking just the last unsatisfiable certificat 5 . From the results we can conclude that as expected checking only the last unsatisfiable certificate can result in significant savings in terms of run times. For example, the normalized-iia81.wcnf becomes two orders of magnitude faster than considering all the certificates. Similar conclusions can be drawn for most of the other benchmarks shown. Nevertheless, some instances show the same time on checking all or just one. This happens on instances with just one unsatisfiable iteration, thus just one unsatisfiable certificate to check. The results allow concluding that the ability to check just one unsatisfiable certificate and one satisfiable certificate can result in important performance improvements when checking the results of Boolean optimization solvers.

\footnotetext{
${ }^{5}$ The running times of checking the satisfiable certificate is negligible and was not considered in either approaches.
} 


\section{Conclusions and Future Work}

This paper investigates solutions for checking the results computed by Boolean optimizers, which are based on iterative calls to a SAT solver. Hence, the paper complements recent work on generating certificates for branch-and-bound Boolean optimization algorithms. The paper overviews all existing algorithms based on iterative calls to a SAT solver, and shows that, for all these algorithms, it suffices to check one unsatisfiability proof and one satisfiable certificate. Experimental results indicate that the overhead of checking the solutions computed by Boolean Optimization algorithms is negligible. Simple implementation improvements to the work described in the paper include eliminating altogether proof tracing, only recreating proof tracing for the (then known) last unsatisfiability proof. This provides additional performance improvements over solutions that might check all unsatisfiability proofs. Additional research work consists in developing solution checking approaches for Max-SMT [26] and Max-ASP [27.

\section{Acknowledgments}

This work is partially supported by SFI PI grant BEACON (09/IN.1/I2618) and by FCT through grant ATTEST (CMU-PT/ELE/0009/2009).

\section{References}

1. Aloul, F., Ramani, A., Markov, I., Sakallah, K.: PBS: A backtrack search pseudoboolean solver. In: Theory and Applications of Satisfiability Testing. pp. 346-353 (2002)

2. Aloul, F.A., Ramani, A., Markov, I.L., Sakallah, K.A.: Generic ILP versus specialized 0-1 ILP: an update. In: Computer-Aided Design. pp. 450-457 (2002)

3. Amgoud, L., Cayrol, C., Berre, D.L.: Comparing arguments using preference ordering for argument-based reasoning. In: Tools with Artificial Intelligence. pp. 400-403 (1996)

4. Ansótegui, C., Bonet, M.L., Levy, J.: Solving (weighted) partial MaxSAT through satisfiability testing. In: Theory and Applications of Satisfiability Testing. pp. 427440 (2009)

5. Ansótegui, C., Bonet, M.L., Levy, J.: A new algorithm for weighted partial maxsat. In: AAAI Conference on Artificial Intelligence (2010)

6. Berre, D.L.: SAT4J library. www.sat4j.org

7. Biere, A., Heule, M., van Maaren, H., Walsh, T. (eds.): Handbook of Satisfiability (2009)

8. Cimatti, A., Franzén, A., Griggio, A., Sebastiani, R., Stenico, C.: Satisfiability modulo the theory of costs: Foundations and applications. In: Tools and Algorithms for the Construction and Analysis of Systems. pp. 99-113 (2010)

9. Eén, N., Sörensson, N.: Translating pseudo-Boolean constraints into SAT. Journal on Satisfiability, Boolean Modeling and Computation 2(3-4), 1-25 (2006)

10. Eén, N., Sörensson, N.: An extensible sat-solver. In: Theory and Applications of Satisfiability Testing. pp. 502-518 (2003) 
11. Fu, Z., Malik, S.: On solving the partial MAX-SAT problem. In: Theory and Applications of Satisfiability Testing. pp. 252-265 (2006)

12. Fu, Z., Malik, S.: Solving the minimum-cost satisfiability problem using SAT based branch and bound search. In: Computer-Aided Design. pp. 852-859 (2006)

13. Goldberg, E.I., Novikov, Y.: Verification of proofs of unsatisfiability for cnf formulas. In: Design, Automation and Test in Europe. pp. 10886-10891 (2003)

14. Heras, F., Larrosa, J., Oliveras, A.: MiniMaxSat: An efficient weighted Max-SAT solver. Journal of Artificial Intelligence Research 31, 1-32 (2008)

15. Heras, F., Morgado, A., Marques-Silva, J.: Core-guided binary search algorithms for maximum satisfiability. In: AAAI Conference on Artificial Intelligence. p. In Press (2011)

16. Jose, M., Majumdar, R.: Cause clue clauses: Error localization using maximum satisfiablity. In: Programming Language Design and Implementation (2011)

17. Larrosa, J., Nieuwenhuis, R., Oliveras, A., Rodríguez-Carbonell, E.: Branch and bound for boolean optimization and the generation of optimality certificates. In: Theory and Applications of Satisfiability Testing. pp. 453-466 (2009)

18. Larrosa, J., Nieuwenhuis, R., Oliveras, A., Rodríguez-Carbonell, E.: A framework for certified boolean branch-and-bound optimization. Journal of Automated Reasoning (2009)

19. Lescuyer, S., Conchon, S.: A reflexive formalization of a SAT solver in Coq. In: Theorem Proving in Higher Order Logics (2008)

20. Li, C.M., Manyà, F.: Maxsat, hard and soft constraints. In: Biere, A., Heule, M., van Maaren, H., Walsh, T. (eds.) Handbook of Satisfiability, vol. 185, pp. 613-631. IOS Press (2009)

21. Manquinho, V., Marques-Silva, J.P.: Satisfiability-based algorithms for Boolean optimization. Annals of Mathematics and Artificial Intelligence 40(3-4) (2004)

22. Manquinho, V., Marques-Silva, J., Planes, J.: Algorithms for weighted Boolean optimization. In: Theory and Applications of Satisfiability Testing. pp. 495-508 (2009)

23. Marques-Silva, J., Manquinho, V.: Towards more effective unsatisfiability-based maximum satisfiability algorithms. In: Theory and Applications of Satisfiability Testing. pp. 225-230 (2008)

24. Marques-Silva, J., Planes, J.: Algorithms for maximum satisfiability using unsatisfiable cores. In: Design, Automation and Test in Europe. pp. 408-413 (2008)

25. Moskewicz, M.W., Madigan, C.F., Zhao, Y., Zhang, L., Malik, S.: Chaff: Engineering an efficient sat solver. In: Design Automation Conference. pp. 530-535 (2001)

26. Nieuwenhuis, R., Oliveras, A.: On SAT modulo theories and optimization problems. In: Theory and Applications of Satisfiability Testing. pp. 156-169 (2006)

27. Oikarinen, E., Järvisalo, M.: Max-ASP: Maximum satisfiability of answer set programs. In: Logic Programming and Nonmonotonic Reasoning. pp. 236-249 (2009)

28. Roussel, O., Manquinho, V.M.: Pseudo-boolean and cardinality constraints. In: Biere, A., Heule, M., van Maaren, H., Walsh, T. (eds.) Handbook of Satisfiability, vol. 185 , pp. $695-733$. IOS Press (2009)

29. Safarpour, S., Mangassarian, H., Veneris, A.G., Liffiton, M.H., Sakallah, K.A.: Improved design debugging using maximum satisfiability. In: Formal Methods in Computer-Aided Design. pp. 13-19 (2007)

30. Sheini, H., Sakallah, K.: Pueblo: A hybrid pseudo-Boolean SAT solver. Journal on Satisfiability, Boolean Modeling and Computation 2(3-4), 165-189 (2006)

31. Weber, T., Amjad, H.: Efficiently checking propositional refutations in hol theorem provers. Journal of Applied Logic 7(1), 26-40 (2009) 
32. Zhang, L., Malik, S.: Validating SAT solvers using an independent resolution-based checker: Practical implementations and other applications. In: Design, Automation and Test in Europe. pp. 10880-10885 (2003) 